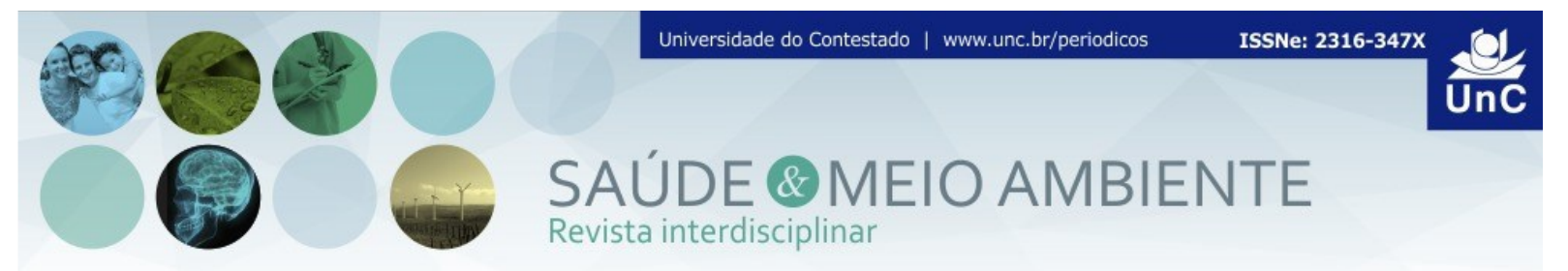

\title{
PERFIL DO CONSUMO DE MEDICAMENTOS POR GRADUANDOS EM UMA UNIVERSIDADE DO PLANALTO NORTE CATARINENSE
}

\section{PROFILE OF DRUG CONSUMPTION BY UNDERGRADUATES IN A UNIVERSITY OF THE NORTH CATHARINIAN PLATEAU}

\author{
Míriam Cristina José Valério ${ }^{1}$ \\ Mayara Morretes ${ }^{2}$
}

\begin{abstract}
RESUMO
Introdução: Segundo a Organização Mundial da Saúde, 2002, a morbidade e a mortalidade causadas por produtos farmacêuticos são problemas de saúde. Objetivo: Este trabalho identificou o hábito da utilização de medicamentos entre estudantes de uma Universidade do Planalto Norte Catarinense, delineando o consumo e seus motivos, para obter subsídios na elaboração da promoção do uso racional de medicamentos. Metodologia: O estudo buscou caracterizar de forma quantitativa os estudantes, foi uma pesquisa descritiva, enfatizando a descrição do consumo de medicamentos, segundo a perspectiva dessa prática enquanto um problema de saúde pública, visando caracterizar o perfil dos medicamentos consumidos e o perfil dos fatores associados. Resultados e discussões: Foram aplicados 234 questionários, sendo $61 \%$ do sexo feminino. A média de idade foi de 17-32 anos. Dos entrevistados, grande parte afirma que não possui nenhum problema de saúde atualmente. Porém, os dados revelam que $57,26 \%$ faz uso de algum medicamento, e que $36,32 \%$ o faz diariamente, sendo a classe de analgésicos é a mais consumida. $14,10 \%$ dos entrevistados sentem-se desanimados antes de consumir o medicamento. Conclusão: o perfil do consumo de medicamentos alerta para a prática da automedicação, desencadeando a necessidade de se realizar estratégias na promoção do uso racional de medicamentos.
\end{abstract}

Palavras-Chave: Uso de medicamentos. Universitários. Automedicação. Emoções.

\section{ABSTRACT}

Introduction: According to the World Health Organization, 2002, the morbidity and mortality caused by pharmaceutical products are health problems. Objective: This study identified the habit of using medications among students at a University of

\footnotetext{
${ }^{1}$ Especialista em Administração Hospitalar e de Clínicas. Universidade do Contestado, Núcleo de Pesquisa em Saúde Coletiva e Meio Ambiente. Coordenadora do Curso de Farmácia da Universidade do Contestado. Campus Mafra. Coordenadora de grupo do PET Saúde Interprofissionalidade. Santa Catarina. Brasil. ORCID: https://orcid.org/0000-0003-4608-867X. E-mail: miriamc@unc.br

${ }^{2}$ Graduanda em Farmácia. Universidade do Contestado, Campus Mafra. Núcleo de Pesquisa em Saúde Coletiva e Meio Ambiente. Bolsista de grupo do PET Saúde Interprofissionalidade. Santa Catarina. Brasil. E-mail: mayara.morretes@aluno.unc.br.
} 
Planalto Norte Catarinense, outlining consumption and its reasons, to obtain subsidies in the elaboration of the promotion of the rational use of medications. Methodology: The study sought to characterize students quantitatively, it was a descriptive research, emphasizing the description of medication consumption, according to the perspective of this practice as a public health problem, aiming to characterize the profile of the drugs consumed and the profile of the associated factors. Results and discussions: 234 questionnaires were applied, $61 \%$ of which were female. The average age was 17-32 years. Of the interviewees, most of them state that they do not currently have any health problems. However, the data reveal that $57.26 \%$ use some medication, and that $36.32 \%$ do it daily, with the analgesic class being the most consumed. $14.10 \%$ of respondents feel discouraged before consuming the medication. Conclusion: the profile of medication consumption alerts to the practice of self-medication, triggering the need to carry out strategies to promote the rational use of medications.

Keywords: Use of medicines. College students. Self-medication. Emotions.

\section{INTRODUÇÃO}

O uso racional de medicamentos está entre os objetivos e diretrizes da Política Nacional de Medicamentos e da Política Nacional de Assistência Farmacêutica. Essa temática vem ganhando expressão ao longo dos últimos anos tanto nacional, quanto internacionalmente. Deixando claro a importância de esclarecer a comunidade sobre os riscos dos medicamentos, de forma imparcial, sem conflito de interesses, favorecendo a promoção do uso racional de medicamentos. Os meios de comunicação, entre outros fatores, acabam por incentivar que os problemas sociais sejam resolvidos pelo uso de medicamentos, passando uma falsa imagem de que isso é bom ${ }^{1}$.

Consumir medicamentos sem prescrição e acompanhamento médico, ou de profissional habilitado, é definido universalmente como automedicação, esta prática pode resultar de uma situação favorável, na melhora dos sintomas do indivíduo, assim como pode agravar problemas de saúde, ao atrasar diagnósticos de doenças ou desencadear intoxicações ${ }^{2,3}$.

O medicamento é um bem essencial à saúde e uma importante ferramenta terapêutica nas mãos dos médicos, sendo responsável por parte significativa da melhoria da qualidade e expectativa de vida da população. Para promover a melhoria das políticas públicas de assistência à saúde do cidadão, é necessário delinear o perfil epidemiológico e os fatores que influenciam o consumo dos medicamentos nas diferentes comunidades ${ }^{4}$. O uso excessivo e incorreto de medicamentos causa danos à saúde e desperdiça recursos.

Verifica-se, ao analisar os dados do Ministério da Saúde, entre 2012 e 2016, no Sistema Nacional de Gestão da Assistência Farmacêutica (Hórus) houve um crescimento e $87 \%$ na dispensação de medicamentos, e no sistema informatizado do Programa Farmácia Popular do Brasil - Rede Própria, houve um aumento de $61 \%$ de 
consumo no mercado total de antidepressivos. Verificando-se que, o medicamento é visto como meio rápido para a resolução de problemas de diversas origens ${ }^{1}$.

Segundo a Organização Mundial da Saúde ${ }^{5}$, durante as últimas décadas, demonstrou-se, por muitos estudos, que a morbidade e a mortalidade causadas por produtos farmacêuticos estão entre os principais problemas de saúde, o que começa a ser reconhecido pelos profissionais de saúde e pelo público. Estima-se que, as reações adversas a fármacos estejam entre a $4^{\mathrm{a}}$ e a $6^{\mathrm{a}}$. maiores causas de mortalidade nos EUA e a cada ano e muitos outros têm sofrimento decorrente de reações adversas.

A percepção de saúde e doença tem sido bastante relacionada ao medicamento. Condições fisiológicas normais em outros tempos, tais como a velhice ou a menopausa, passam a ser vistas como doença. Esse processo é chamado de "medicalização da vida" e tem como consequência inicial o aumento do mercado consumidor de medicamentos ${ }^{6,7}$. Esse aumento do consumo de medicamentos é favorável à Indústria, no entanto, o uso indiscriminado de medicamentos pode oferecer risco à saúde da população.

A cultura da medicalização se instala numa sociedade quando se busca maior rendimento no trabalho, maior produtividade, melhoria na estética corporal, sem perceber a real noção dos riscos do tratamento farmacológico, e até mesmo da dependência física ou psíquica intrínseca no uso excessivo desses medicamentos, podendo assim, ser considerado algo patológico ${ }^{8}$.

Os estudantes universitários formam uma comunidade jovem e potencialmente saudável, o seu hábito de consumo de medicamentos, provavelmente não reflete o hábito da população em geral. No entanto, estão expostos a fatores de risco específicos, nomeadamente um contínuo stress e esforço intelectual intenso, sua rotina árdua associada a um ambiente acadêmico nem sempre saudável, pode favorecer o desenvolvimento de transtornos psíquicos, o que pode ocorrer entre 15 a $25 \%$ dos estudantes ${ }^{9,10}$. Estes fatores, favorecem que o jovem universitário seja um potencial consumidor de medicamentos.

Por serem os alunos universitários, potenciais líderes de opinião, a caracterização do padrão de consumo de medicamentos neste grupo, não só pode contribuir para um melhor conhecimento sobre a sua saúde, mas também para a elaboração de programas que visem o uso racional do medicamento na população em geral $^{10}$. É importante ressaltar que, uma das principais justificativas encontradas para a automedicação, é justamente a facilidade de tratamento disponibilizada por um maior acesso aos fármacos do que aos serviços de saúde ${ }^{11}$.

Se faz necessário a mobilização da comunidade científica na promoção da saúde, estimulando a diminuição do consumo dos medicamentos, ao esclarecer que medicamento é um bem essencial à saúde e uma importante ferramenta terapêutica, mas que seu uso irracional eleva não somente os gastos na área da saúde, como pode afetar diretamente a saúde da população. Assim, este trabalho tem por objetivo Investigar os medicamentos consumidos pelos universitários de uma universidade do Planalto Norte Catarinense, Citar os tipos de medicamentos mais consumidos; 
Descrever os motivos que levam ao consumo de medicamentos; Reconhecer qual a orientação que o levou a consumir a medicação; Produzir material educativo para o consumo consciente dos medicamentos.

\section{MATERIAL E MÉTODOS}

A pesquisa se caracterizou por um estudo transversal, observacional e descritivo. O método utilizado para coleta de dados foi uma abordagem quantitativa, que utilizou de técnicas estatísticas para quantificar o modo de coleta e tratamento dos dados. Conforme Marconi e Lakatos ${ }^{12}$, este método de pesquisa tem o propósito adquirir informações e/ou conhecimentos em relação a um dado problema para o qual se busca uma resposta, comprovação de uma hipótese ou descoberta de novos fenômenos e relações entre estes.

Esta pesquisa foi aprovada pelo Comitê de Ética em Pesquisa, da Universidade do Contestado, obtendo parecer favorável através do número 3.669.041.

Os participantes desta pesquisa foram acadêmicos da instituição de ensino Universidade do Contestado, sendo eles matriculados nos cursos de Administração, Arquitetura e Urbanismo, Ciências Biológicas, Ciências Contábeis, Direito, Educação Física, Enfermagem, Engenharia de Software, Farmácia e Psicologia. Estimou-se, pela análise de cálculo amostral entrevistar no mínimo 315 acadêmicos, usando o nível de confiança de $95 \%{ }^{13}$.

É estimado o aceite de aproximadamente 221 participantes, considerando uma perda de $30 \%$ da amostra. Após a ciência dos participantes e a assinatura do Termo de Consentimento Livre e Esclarecido (TCLE), os participantes foram convidados a realizar o preenchimento do instrumento, através de um formulário no Google Docs, para a obtenção dos dados que foram então compilados e organizados para análise.

A coleta de dados se deu através de uma entrevista estruturada, a qual teve como característica o uso de um roteiro previamente definido. As questões e respostas objetivas foram antecipadamente codificadas para facilitar a tabulação de dados. Questionário é alinhado a uma série de questões que devem ser respondidas pelo indivíduo que detêm as informações que serão pertinentes a pesquisa, deve conter instruções que ressaltem a necessidade de uma execução correta ${ }^{13}$.

As variáveis estudadas foram os dados de formação dos estudantes, como curso e fase, sua idade, como sendo variáveis independentes. Sendo analisadas as variáveis dependentes como, trabalhar, manifestar doença, consumir medicamentos e suas motivações para o consumo. Considerando para isso, que variável independente é aquela que influencia outra variável e dependente o fenômeno a ser explicado $^{12}$. Após compilados os dados foram analisados por meio de análise estatística com cálculo de estatística descritiva e das frequências relativas e absolutas. 


\section{RESULTADOS}

Inicialmente foi identificado o perfil do graduando em uma universidade norte do Planalto Catarinense. Foram aplicados 234 questionários houve com predominância do sexo feminino 142 (61\%). A média de idade foi de 17-32 anos, com o mínimo de 17 anos e o máximo de pessoas com mais de 32 anos. Em relação ao curso de graduação observa-se uma média de 20 alunos por turma exceto o curso de Direito que possui mais de 50 alunos. A questão se está trabalhando atualmente, cerca de $179(76,50 \%)$ dos entrevistados afirmou que sim e em relação a algum problema de saúde no momento, $144(61,53 \%)$ dos entrevistados afirmaram que não. Entre os entrevistados, grande parte afirma que não possui nenhum problema de saúde atualmente, entretanto $n=89(38,03 \%)$ afirma que possui. (Tabela 1$)$

Tabela 1 - Perfil da população acadêmica estudada, como gênero, faixa etária, curso, se está trabalhando e se apresenta algum problema de saúde atualmente, dados coletados do município de Mafra SC, em novembro de 2019

\begin{tabular}{|c|c|c|}
\hline Variáveis & $\mathbf{N}$ & $\%$ \\
\hline \multicolumn{3}{|c|}{ Sexo } \\
\hline Feminino & 142 & 61,00 \\
\hline Masculino & 91 & 39,00 \\
\hline \multicolumn{3}{|l|}{ Idade (Em anos) } \\
\hline 17 a 21 anos & 106 & 45,29 \\
\hline 21 a 23 anos & 74 & 31,62 \\
\hline 24 a 27 anos & 24 & 10,25 \\
\hline 28 a 31 anos & 11 & 4,70 \\
\hline 32 anos ou mais & 18 & 7,70 \\
\hline \multicolumn{3}{|c|}{ Fase } \\
\hline $1^{a}$ Fase & 1 & 0,42 \\
\hline $2^{a}$ Fase & 71 & 30,35 \\
\hline $3^{a}$ Fase & 3 & 1,28 \\
\hline $4^{a}$ Fase & 42 & 18,00 \\
\hline $5^{a}$ Fase & 1 & 0,42 \\
\hline $6^{a}$ Fase & 49 & 21,00 \\
\hline $8^{a}$ Fase & 40 & 17,09 \\
\hline $10^{a}$ Fase & 26 & 11,11 \\
\hline \multicolumn{3}{|c|}{ Curso } \\
\hline Administração & 20 & 8,54 \\
\hline Arquitetura e Urbanismo & 14 & 5,98 \\
\hline Ciências Biológicas & 23 & 9,82 \\
\hline Ciências Contábeis & 22 & 9,40 \\
\hline Direito & 54 & 23,07 \\
\hline Educação Física & 16 & 6,84 \\
\hline Enfermagem & 24 & 10,25 \\
\hline Engenharia de Software & 13 & 5,55 \\
\hline Farmácia & 23 & 9,82 \\
\hline Psicologia & 24 & 10,25 \\
\hline \multicolumn{3}{|c|}{ Está trabalhando? } \\
\hline Sim & 179 & 76,50 \\
\hline Não & 54 & 23,07 \\
\hline \multicolumn{3}{|c|}{ Você apresenta algum problema de saúde atualmente? } \\
\hline Sim & 89 & 38,03 \\
\hline Não & 144 & 61,53 \\
\hline
\end{tabular}

Fonte: Dados da pesquisa (2019) 
Sobre o uso de medicamentos, os dados revelam que $n=136(57,26 \%)$ faz uso de algum medicamento, e que $n=85$ (36,32\%) o faz diariamente. Ao analisar os dados, destaca-se o grande consumo de analgésicos onde $n=69(29,48 \%)$ fazem o desta classe principalmente.

Os universitários em grande parte aderem a orientação médica, aproximadamente, $n=112(49,9 \%)$ seguem a indicação deste profissional. Pode-se observar que $n=122(52,9 \%)$, buscam outras orientações, como a própria família ou farmacêutico.

Tabela 2 -Sobre o Uso do medicamento

\begin{tabular}{|c|c|c|}
\hline Variáveis & $\mathbf{N}$ & $\%$ \\
\hline \multicolumn{3}{|c|}{ Faz uso de algum medicamento? } \\
\hline $\operatorname{Sim}$ & 136 & 57,26 \\
\hline Não & 97 & 41,45 \\
\hline \multicolumn{3}{|c|}{ Se sim, qual a frequência do consumo? } \\
\hline Diário & 85 & 36,32 \\
\hline Eventualmente & 28 & 12,00 \\
\hline Semanal & 6 & 2,56 \\
\hline Mensal & 17 & 7,26 \\
\hline \multicolumn{3}{|c|}{ Qual a classe de medicamentos que você mais utiliza? } \\
\hline Analgésico & 69 & 29,48 \\
\hline Antialérgicos & 6 & 2,56 \\
\hline Antibióticos & 7 & 2,99 \\
\hline Anticoncepcional & 19 & 8,11 \\
\hline Anti-inflamatórios & 6 & 2,56 \\
\hline Antitérmicos & 6 & 2,56 \\
\hline Estimulantes & 4 & 1,70 \\
\hline Psicotrópicos (calmantes, antidepressivo, rivotril, fluoxetina) & 12 & 5,12 \\
\hline Associação entre 2 ou mais medicamentos & 98 & 41,55 \\
\hline \multicolumn{3}{|c|}{ Quem indicou o uso do medicamento? } \\
\hline Indicação própria & 9 & 3,84 \\
\hline Orientação Médica & 112 & 47,9 \\
\hline Orientação Familiar & 41 & 17,52 \\
\hline Orientação Farmacêutica & 31 & 13,24 \\
\hline Orientação Farmacêutica, Familiar & 8 & 3,41 \\
\hline Orientação Médica, Familiar & 7 & 2,99 \\
\hline Orientação Médica, Farmacêutica & 9 & 3,84 \\
\hline Orientação Médica, Enfermeira & 1 & 0,42 \\
\hline Orientação Médica, Farmacêutica e Familiar & 6 & 2,56 \\
\hline Internet & 1 & 0,42 \\
\hline N.D.A & 7 & 2,99 \\
\hline
\end{tabular}

Fonte: Dados da pesquisa (2019)

Em relação às emoções ligadas ao consumo de medicamentos, foi observado que $n=33(14,10 \%)$ dos entrevistados sentem-se desanimados, $n=23(9,82 \%)$ sentemse chateados e $n=44(18,80 \%)$ consideram a raiva como uma emoção presente no seu dia-a-dia. Entretanto, vale observar que $n=81(34,61 \%)$ dos entrevistados relatam alegria como uma emoção presente em seu dia-a-dia. 
Tabela 3 - Emoções ligadas ao consumo de medicamentos

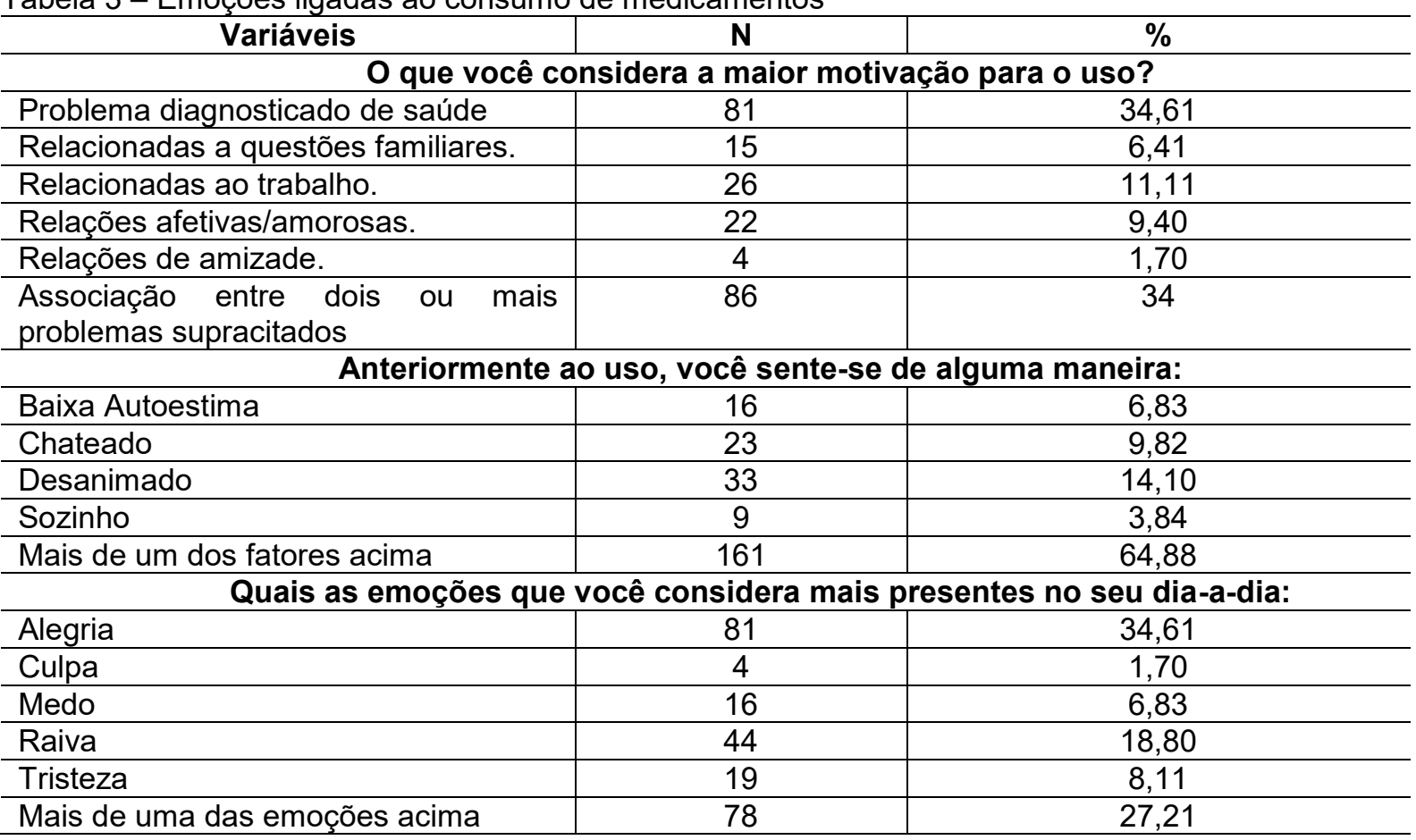

Fonte: Dados da pesquisa (2019)

\section{DISCUSSÕES}

Através dos dados analisados, pode-se perceber que o gênero dos estudantes é compatível aos estudantes de ensino superior no Brasil, conforme a publicação do Censo da Educação Superior 2018, o qual relata a predominância do sexo feminino em relação ao número de estudantes matriculados nas modalidades de ensino presencial e a distância ${ }^{1}$.

O estudo demonstrou que os estudantes participantes da pesquisa, relataram não possuir problema de saúde, identificando sua saúde como boa ou muito boa. Porém, esta percepção pode não traduzir sua real condição de saúde, visto que, a Organização Mundial de Saúde (OMS) define saúde como um estado pleno de desenvolvimento mental, físico e de bem-estar social, não sendo meramente a ausência de doença ${ }^{5}$. De acordo os dados informados pelo IBGE, 81,6\% dos jovens brasileiros, entre 18 e 29 anos apresentam a percepção de sua própria saúde como boa ou muito boa ${ }^{14}$.

Segundo Gomes et al. ${ }^{15}$, quase metade dos adultos brasileiros consomem algum medicamento, reforçando os dados identificados. O consumo de medicamentos está associado ao risco de intoxicação pelo mesmo, conforme o Centro de Informações e Assistência Toxicológicas de Santa Catarina ${ }^{16}$, 25,9\% dos casos de intoxicação em Santa Catarina são pelo uso indevido de medicamentos, seja acidental ou não. 
Identificar pelo estudo, que os analgésicos, são a principal classe de medicamento mais consumida, corrobora os resultados de outras pesquisas. Conforme cita Cariman et al. ${ }^{17}$ em seu estudo bibliográfico, entre os fármacos mais utilizados pelos universitários encontram-se os analgésicos (36\%). Isto se deve ao fato de serem usados no alívio de qualquer processo de dor, por possuírem fácil aquisição e em grande parte por apresentarem venda livre, não necessitando de receita médica.

Os fármacos analgésicos, segundo a Resolução no. 138/03, são Medicamentos Isentos de Prescrição (MIP), sendo considerados medicamentos indicados para tratar pequenos males que não necessitam de prescrição médica. No entanto, se utilizados em excesso e sem real necessidade podem acarretar em efeitos nocivos à saúde como insuficiência renal, doenças hepáticas, ou mascarar sintomas de certas enfermidades, entre outros problemas ${ }^{18}$. Em consonância com este dado, a Academia Brasileira de Neurologia ${ }^{19}$, realizou uma pesquisa, revelando que $80 \%$ da população brasileira toma medicamentos analgésicos para dor de cabeça sem orientação médica, o que pode atrapalhar o tratamento correto para a cefaleia.

A análise das informações, demonstrou que os estudantes têm por hábito seguir a orientação médica, mas muitos ainda buscam orientações do farmacêutico ou com familiares. Estes dados são inferiores a outros estudos, Cruz et al. ${ }^{20}$, verificou que a prática da automedicação entre estudantes universitários se dá em $78 \%$ da população entrevistada. Galato et al. ${ }^{3}$, identificou que $96 \%$ dos estudantes, na faixa etária de 22,9 anos, já se automedicaram. Já Fontanella et al. ${ }^{21}$, apresenta um dado alarmante em sua pesquisa, revelando que $52 \%$ dos entrevistados, com idade média de 24,5 anos haviam se automedicado na semana anterior a pesquisa.

Ao correlacionar as emoções ligadas ao consumo de medicamentos, é possível perceber que os estudantes consomem medicamentos motivados principalmente por pela presença de algum problema de saúde, mas também manifestam outras motivações, entre as quais foi possível destacar questões relacionadas ao trabalho, família, amizade e amorosas, sugerindo que este público enfrenta algum tipo de sofrimento no período de sua graduação, além das dificuldades já inerentes à graduação. Entretanto, vale observar que muitos dos entrevistados relataram alegria como uma emoção presente em seu dia-a-dia.

Estas emoções e sensações, são frequentemente identificadas em outros estudos. Um estudo realizado por Souza et al. ${ }^{22}$ na Universidade Federal do Triangulo Mineiro, com 384 estudantes, identificou a presença de sofrimento psíquico neste público, sendo que $47 \%$ afirmaram sentimento de solidão, $59,2 \%$ dos participantes relataram sintomas depressivos, $70,4 \%$ com prevalência de ansiedade e $78,1 \%$ apresentaram estresse. Uma série de demandas características do contexto universitário está associado ao sofrimento mental dos estudantes, podendo citar necessidade de adaptação, suporte social, dificuldades com os conteúdos abordados, entre outras ${ }^{23}$.

Conforme Soares ${ }^{24}$, ao estabelecer relações interpessoais com demais colegas e professores, o aluno consegue vivenciar mais tranquilamente a universidade. Assim 
como, faz parte das atribuições das instituições, favorecer ao aluno a aquisição de habilidades e competências necessárias para exercer sua profissão.

\section{CONCLUSÃO}

Ao avaliar o hábito de consumo de medicamentos, pode-se observar que a maior parte dos entrevistados consome algum medicamento diariamente, seja por questões associadas a algum problema específico de saúde ou por questões relacionadas ao trabalho, família ou a vida afetiva.

Percebe-se que a população estudada apresenta um consumo considerado baixo em relação a outros jovens estudados ${ }^{20}$. Sendo que, a classe de medicamentos mais consumida é a dos analgésicos, refletindo o consumo da população brasileira.

Muitos fatores podem levar ao consumo de medicamentos, podendo desencadear assim, outros problemas de saúde, especialmente, quando não se trata a causa, mas sim o sintoma do problema de saúde percebido. Os fatores mais apontados para o consumo de medicamentos foram por problemas de saúde ou com o trabalho, prevalecendo o consumo através de orientação médica.

A maioria dos medicamentos consumidos, são isentos de prescrição, mas não isentos de risco, o que sugere a necessidade de estratégias para a promoção da saúde, que podem ser adotadas de forma interprofissional, levando orientação efetiva sobre automedicação e seus riscos potenciais, aumentado a eficiência nos resultados obtidos. Demostrando a importância de se tratar deste tema com mais atenção. É importante considerar outras pesquisas para se aprofundar no entendimento do tema e dos dados apresentados.

\section{REFERÊNCIAS}

1 Brasil. Ministério da Saúde. Secretaria de Vigilância em Saúde. Departamento de Vigilância de Doenças e Agravos não Transmissíveis e Promoção da Saúde. Saúde Brasil 2018 uma análise de situação de saúde e das doenças e agravos crônicos: desafios e perspectivas. Brasília: Ministério da Saúde; 2019. [acesso em 2019 Jun 12]. Disponível em: http://bvsms.saude.gov.br/bvs/publicacoes/ saude_brasil_2018_analise_situacao_saude_doencas_agravos_cronicos_desafio s_perspectivas.pdf.

2 Locquet M, Honvo G, Rabenda V, Van Hees T, Petermans J, Reginster JY, et al. (2017). Adverse health events related to self-medication practices among elderly: a systematic review. Drugs Aging. 2017; 34(5):359-65. Review. 
3 Galato D, Madalena J, Pereira G. B. Automedicação em estudantes universitários: a influência da área de formação. Ci. Saúde Col. 2012;17(12):3323-3330.

4 Arrais P S D, Brito L L, Barreto M L, Coelho H L L. Prevalência e fatores determinantes do consumo de medicamentos no Município de Fortaleza, Ceará, Brasil. Cad. Saúde Pública [Internet]. 2005 Dec [acesso em 2019 set 10] ; 21( 6 ): 1737-1746. Disponível em: http://www.scielo.br/scielo.php?script= sci_arttext\&pid=S0102-311X2005000600021\&lng=en.

5 World Health Organization. The World health report: 2002: Reducing the risks, promoting healthy life. World Health Organization; 2002. Disponível em: https://apps.who.int/iris/handle/10665/4251

6 Sobravime (Sociedade Brasileira de Vigilância de Medicamentos). O que é uso racional de medicamentos. Acción Internacional para la Salud: América Latina y El Caribe. São Paulo: Sobravime; 2001.

7 Dasilva MVS; Mendes IJM; Freitas O. O medicamento, a auto-medicação e a farmácia. Infarma 2002;15(3/4).

8 Brasil. Ministério da Saúde. Secretaria de Ciência, Tecnologia e Insumos Estratégicos. Departamento de Assistência Farmacêutica e Insumos Estratégicos. Uso de Medicamentos e Medicalização da Vida: recomendações e estratégias. Brasília : Ministério da Saúde; 2018.

9 Sakae TM, Padão DL, Jordana LK. Sintomas depressivos em estudantes da área da saúde em uma universidade no sul de Santa Catarina - UNISUL. Revista da Associação Médica do Rio Grande do Sul 2010; 54(1):38-43.

10 Cabrita J, Ferreira, H, Iglésias, P, Baptista T, Rocha E, Silva AL, Miguel JP. Estudo do padrão de consumo de medicamentos pelos estudantes da Universidade de Lisboa. Revista Portuguesa de Saúde Pública, 2001 jul./Dez.; 19(2):39-47.

11 Silva J A C., Gomes A L, Oliveira J P S, Sasaki Y A, Maia B T B, Abreu B M Prevalência de automedicação e os fatores associados entre os usuários de um Centro de Saúde Universitário. Rev Bras Clin Med. São Paulo, 2013 jan-mar; 11(1):27-30.

12 Marconi M A, Lakatos E M. Fundamentos de metodologia cientifica. 7. ed. São Paulo: Atlas; 2010.

13 Matias-Pereira J. Manual de metodologia da pesquisa científica. 3. ed. São Paulo: Atlas; 2012.

14 Instituto Brasileiro de Geografia e Estatística. Coordenação de Trabalho e Rendimento. Pesquisa Nacional de Saúde 2013: percepção do estado de saúde, estilos de vida e doenças crônicas. Brasília: Ministério de Planejamento, Orçamento e Gestão, Instituto Brasileiro de Geografia e Estatística-IBGE, Diretoria de Pesquisas, Coordenação de Trabalho e Rendimento; 2014. 
15 Gomes V P, Silva M T, Galvão T F. Prevalência do consumo de medicamentos em adultos brasileiros: uma revisão sistemática. Ciênc. Saúde Coletiva 2017 ago; 22 (8): 2615-2626.

16 Universidade Federal de Santa Catarina. Hospital Universitário Polydoro Ernani de São Thiago. Centro de Informação e Assistência Toxicológica de Santa Catarina: Relatório Anual 2015. Florianópolis, SC: HU/UFSC; 2016.

17 Cariman L I C, Silva R I, Sousa J B B. Estudos da utilização de medicamentos pela população universitária. Revista Interdisciplinar de Ciências Médicas: Anais. Teresina, PI.

18 Silva A F, Rodrigues AM. Automedicação entre estudantes de cursos da área de saúde. Revista Brasileira Farmacologia 2014; 95 (3): 961-975.

19 Academia Brasileira de Neurologia. Pesquisa da ABN sobre enxaqueca expõe o grave quadro de automedicação no Brasil; 2017 maio 16. [Acesso em: 26 jan. 2020]. Disponível em: http://www.abneuro.org.br/clippings/detalhes/842/pesquisada-abn-sobreenxaqueca-expoe-o-grave-quadro-de-automedicacao-no-brasil.

20 Cruz E S, Silva I, Augusto V, Coelho A. Incidência da automedicação entre jovens universitários da área da saúde e de humanas. Rev Saude UniToledo 2019 abr.; $3(1): 2-12$.

21 Fontanella F G; Galato D; Remor K V T. Perfil de automedicação em universitários dos cursos da área da saúde em uma instituição de ensino superior do sul do Brasil. Rev. Bras. Farm. 2013; 94(2): 154-160.

22 Souza D C. Condições emocionais de estudantes universitários: estresse, depressão, ansiedade, solidão e suporte social. Uberaba. Dissertação [Mestrado em Psicologia] - Programa de Pós-Graduação em Psicologia, Universidade Federal do Triângulo Mineiro; 2017.

23 Padovani R C, Neufeld C B, Maltoni J, Barbosa L N F, Souza W F, Cavalcanti H A F, Lameu J N. Vulnerabilidade e bem-estar psicológicos do estudante universitário. Revista Brasileira de Terapias Cognitivas 2014; 10(1), 02-10. Doi: 10.5935/1808- 5687.20140002

24 Soares A B, Porto A M, Lima C A, Gomes C, Rodrigues D A, Zanoteli R, Santos Z A, Fernandes A, Medeiros H. Vivências, Habilidades Sociais e Comportamentos Sociais de Universitários. Psic.: Teor. e Pesq. 2018; 34: e34311. [Acesso em 27 Feb. 2020]. Disponível em: http://www.scielo.br/scielo.php?script=sci_ arttext\&pid=S0102-37722018000100410\&Ing=en\&nrm=iso>. 
AGRADECIMENTO: Agradecemos ao Programa de Bolsas Universitárias se Santa Catarina / UNIEDU, que proveu via artigo 170 as bolsas dos pesquisadores.

Artigo recebido em: 10/03/2020

Artigo aprovado em: $16 / 11 / 2020$

Artigo publicado em: 02/12/2020 